\title{
Evolution of syphilis incidence in Sibiu county (Romania) over a period of 10 years (2009-2018)
}

\author{
GABRIELA MARIANA IANCU ${ }^{1,2}$ and MARIA ROTARU ${ }^{1,2}$ \\ ${ }^{1}$ Department of Dermatology, Lucian Blaga University of Sibiu, Faculty of Medicine, 550169 Sibiu; \\ ${ }^{2}$ Clinic of Dermatology, County Emergency Hospital of Sibiu, 550245 Sibiu, Romania
}

Received March 3, 2021; Accepted April 2, 2021

DOI: $10.3892 / \mathrm{etm} .2021 .10235$

\begin{abstract}
Syphilis is the most common sexually transmitted disease that mainly affects socially active people, with a fluctuating worldwide incidence over the years. A retrospective study was conducted over a period of 10 years (2009-2018) that included 396 patients with syphilis diagnosed and monitored by Sibiu County Emergency Clinical Hospital, Romania. During this period (2009-2018), we observed a decrease in the new cases of syphilis by $35.90 \%$, and the average syphilis incidence was 9.90 new cases per 100,000 inhabitants. Syphilis was diagnosed more frequently in men with an average age of 35.39 years, from urban areas, with and educational level of grades 9-12, unmarried, workers, or without occupation. Out of the 396 patients with syphilis, about $6 \%$ were HIV coinfected. Over half of the HIV coinfected cases were declared MSM (men who have sex with men), and $40 \%$ of these cases were registered in 2018. The most common clinical stage of syphilis was the latent form (67.93\%). Regarding the syphilis treatment regimen, we noted the transition from the classic regimen with benzathine-penicillin G (100\% in 2009) to alternative therapies (mainly with cephalosporins) in 2018 (56.86\%). Our findings showed that in the last 10 years in our county, the incidence of syphilis had a downward trend, but with an increase in syphilis-HIV co-infection and neurosyphilis cases.
\end{abstract}

Correspondence to: Dr Gabriela Mariana Iancu, Department of Dermatology, Lucian Blaga University of Sibiu, Faculty of Medicine, 2A Lucian Blaga Avenue, 550169 Sibiu, Romania

E-mail:mgabiancu@yahoo.com

Abbreviations: HIV, human immunodeficiency virus; MSM, men who have sex with men; BPG, benzathine-penicillin G; STR, serologic treatment response; VDRL, venereal disease research laboratory; RPR, rapid plasma reagin; SD, standard deviation; NIPH, National Institute of Public Health

Key words: syphilis, sexual behavior, human immunodeficiency virus

\section{Introduction}

Syphilis is the most common sexually transmitted disease whose worldwide incidence fluctuates annually or per decade. Although syphilis is a curable disease with accessible treatments and diagnostic tests, it still represents a serious public health problem (1). Syphilis, the great imitator, which can mimic many dermatological conditions (psoriasis, pityriasis rosea, parapsoriasis, drug reactions, lichen planus, sarcoidosis, infectious mononucleosis, lymphoma, and others) $(1,2)$ can have various clinical aspects, from classical to particular forms (seronegative primary syphilis, syphilitic balanitis without a chancre, ocular syphilis) (3-5). Worldwide, about 6 million new cases of syphilis per year are reported, with a mortality of about 5\% in the neonatal or fetal period (6). International prevention programs for sexually transmitted diseases have focused on 4 diseases (syphilis, gonorrhea, trichomoniasis and Chlamydia infections). The target of these programs for 2030 is to reduce the overall incidence of infections; to decrease congenital syphilis to $<50$ cases per 100,000 newborns and the incidence of syphilis by $90 \%$ (7).

In the last decade, the increase in syphilis incidence is based on unrestricted sexual behavior with unprotected contact between multiple partners (8), including the same sex. In 2017, the USA reported the highest rate of syphilis in the last 15 years (9), the data being similar to some European countries.

The high incidence of human immunodeficiency virus (HIV) infection, especially in the MSM (men who have sex with men) category, is also correlated with an increase in syphilis incidence. Syphilis-HIV co-infection is higher in MSM. Karp et al estimate that the risk of syphilis-HIV co-infection is 8 times higher in this category of patients (10).

In September 2019 at the International Union Against Sexually Transmitted Infections (IUSTI) Conference in Tallinn, Estonia, the latest results of the reported syphilis incidence in Europe were announced. Georgia (33 cases per 100,000 inhabitants) had the highest incidence, followed by Malta (18.6 cases per 100,000 inhabitants), Russia (16.8 cases per 100,000 inhabitants), UK (13.6 cases per 100,000 inhabitants) and Switzerland (10.7 cases per 100,000 inhabitants) (11).

Available data for Romania rank it among the countries with less than 5 cases per 100,000 inhabitants (11). In Romania, between 2007 and 2017, there was a downward trend 
in the incidence of syphilis from 20.1 to 4.1 cases per 100,000 inhabitants (12). This decrease may not be factual and may be due to the elimination of certain filters that detect the luetic infection. Currently, it is mandatory to perform these tests only in marriage, pregnancy, blood donation and exposure to a syphilis-infected person.

For proper assessment, it is necessary to implement intensive population education programs, reintroduce the obligation to perform specific serological investigations (at employment or changing jobs), and actively detect latent, atypical and asymptomatic syphilis cases. A delicate topic is represented by untreated mothers infected with Treponema pallidum diagnosed late in pregnancy or at birth (due to unregular check-ups during pregnancy). Their newborns have a high risk of developing congenital syphilis. In addition, the lack of benzathine-penicillin G (BPG) on the Romanian pharmaceutical market has driven doctors to adopt alternative treatment regimens with some oral antibiotics having a possible lower efficacy than the classic schemes.

Our primary objective was to analyzed the evolution of syphilis incidence in the last decade in Sibiu County, and we correlated the results with national and international data. Our secondary objectives were to correlated the demographic data with clinical forms, disease stages, the serologic treatment response and treatment followed by patients with syphilis, over a period of 10 years (2009-2018), in Sibiu County, Romania.

\section{Patients and methods}

Sample selection criteria. We conducted a retrospective study over 10 years (from January 2009 to December 2018) which included 396 patients with syphilis diagnosed, monitored, and treated at the Dermato-Venerological Division of the Clinical Hospital of Sibiu. In our group of patients, we analyzed: the syphilis incidence, demographic data (sex, background, marital status, age group, level of education and occupation), the sources of infection and the number of declared contacts, the evolution of syphilis-HIV co-infection cases, the disease stages and the followed treatment schemes. To identify the sources of infection, the patients answered questions regarding their sexual partners and the prevention methods that they used. Epidemiological and clinical data were included in a personal file. All the patients were tested positive for non-treponemal [venereal disease research laboratory (VDRL) or rapid plasma reagin (RPR)] and treponemal tests [Treponema pallidum hemagglutination assay (TPHA)]. Non-treponemal tests (VDRL or RPR) were used to determine the serologic treatment response after 6 months and 1 year from diagnosis.

Statistical analysis. We analyzed the venerology records of 396 patients diagnosed with syphilis in the last 10 years in Sibiu County. Statistical analysis was performed using SPSS statistics software (version 25.0; IBM Corp.) with alpha $(\alpha)=0.05$ and $\mathrm{CI}$ (confidence interval) $=95 \%$. The variables are expressed in numbers and percentages. The results are presented as median \pm standard deviation (SD). To assess the statistical significance, we calculated the P-value using the Kolmogorov-Smirnov test. P-value $<0.05$ was considered as indicative of statistically significance.
Statement of ethics. The clinical study received the approval of the Ethics Commission of the Sibiu County Clinical Hospital (no. 25413/05.12.2019).

\section{Results}

During the years 2009-2018 in Sibiu county (average population of 400,000 inhabitants), the average syphilis incidence was 9.90 new cases per 100,000 inhabitants. In this period, the incidence of syphilis decreased from a maximum of 19.5 new cases/100,000 (in 2009) to a minimum of 5.25/100,000 (in 2014), and then increased to $12.75 / 100,000$ (in 2018) (Fig. 1).

The average age $( \pm \mathrm{SD})$ of the individuals diagnosed with syphilis was $35.39 \pm 14.64$ years, and $76.52 \%$ belonged to the age group of 15-45 years. The majority of the syphilis patients were men (54.29\%), from urban areas $(67.42 \%)$, with a degree of schooling of grades 9-12 (51.01\%), unmarried (41.92\%), worker $(34.60 \%)$ or unemployed $(32.32 \%)$ (Table I).

Out of the 396 syphilis patients, 25 were HIV coinfected $(6.31 \%)$. In this category of patients, over $50 \%$ declared that they were MSM (56\%), 40\% were heterosexual, and $4 \%$ were female sex workers. In this group of patients (syphilis-HIV co-infection), an ascending progressive distribution was found; $40 \%$ of these cases were confirmed in 2018 (Fig. 2).

Analyzing the syphilis stages, we observed that early (35.61\%) and late latent syphilis (32.32\%) were most common (Fig. 3) $(\mathrm{P}<0.001)$.

Regarding the detection method, over $1 / 3$ of the cases were diagnosed by epidemiological investigation (39.65\%), respectively by investigating all the declared contacts of a new case of syphilis. Other detection methods were: unspecified (32.58\%), pregnancy/birth (14.39\%), blood donation (6.82\%), tests performed at employment $(4.80 \%)$ and prenuptial assessment $(1.77 \%)(\mathrm{P}<0.001)$. From the 396 syphilis patients, 276 contacts were declared by epidemiological investigation, and 183 contacts out of the 276 were found, the rest being accidental relationships with unknown people.

Regarding the syphilis treatment scheme, the Health Ministry's indications (adapted to each stage) were respected. In the last decade, there was a transition from the classic scheme with BPG/penicillin (100\% in 2009) to alternative therapies, mainly with cephalosporins in 2018 (56.86\%) $(\mathrm{P}<0.001)$ (Table II).

The serologic treatment response was evaluated at 6 months and 1 year, according to syphilis stages. The serologic treatment response (STR) was relatively similar in all treatment groups (56.32\% for BPG/ penicillin; $61.11 \%$ for cephalosporins and $72.73 \%$ for doxycycline). Out of all of the groups, the azithromycin/erythromycin had the lowest STR (50\%).

\section{Discussion}

In Sibiu County, from January 2009 to December 2018, the overall incidence of syphilis decreased from 19.75 new cases/100,000 inhabitants in 2009 to 5.25/100,000 in 2014 but reached $12.75 / 100,000$ in 2018. There was a decrease of $35.90 \%$ per decade if we compare the year 2009 with 2018. From January 2009 to December 2018, the average syphilis incidence in our county was 9.90/100,000 inhabitants, above the national average $(4.1 / 100,000$ in 2017) (12). The 


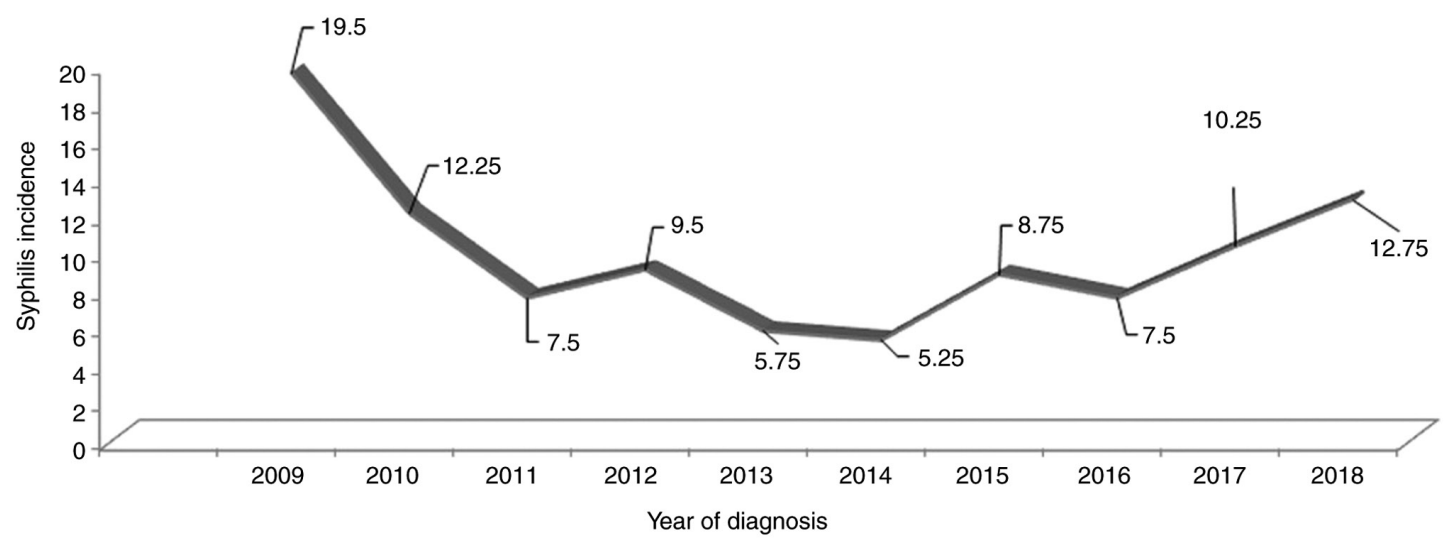

Figure 1. The average syphilis incidence $(9.90$ new cases/100,000) over a period of 10 years (2009-2018) in Sibiu County.

downward trend of the average incidence of syphilis in our county is in accordance with the national data $(20.1 / 100,000$ in 2007 to $4.1 / 100,000$ in 2017) (12), but in discordance with the European data (12). An incomplete report of the new cases of syphilis by the private offices can be considered to explain these results.

The European Center for Disease Prevention and Control analyzed the data that were reported by 30 European countries in 2010-2017 and observed a significant increase in the incidence of syphilis (between 123 and 876\%) in some European countries (Iceland, Ireland, UK, Germany, and Malta) and a decrease of over $50 \%$ in other countries, including Romania (13). Furthermore, in the US, after the year 2000, the incidence of syphilis increased in both men and women (6). The largest increase in luetic infection is cited by Chen et al in China, where after 1970, in 25 years, the syphilis incidence increased by $250-1,000$ times (14).

In our study group, the frequency of luetic disease was higher in men (54.29\%), with a male-to-female ratio of 1.18:1, similar to that reported nationally (below 2:1) (12). Globally, men have a 6-8 times higher risk of having syphilis than women. In Europe in 2013, the M:F ratio was 8.4:1.6, and in the US, it was 15.6:1.9 in 2016; the risk being higher for MSM (6). In our study, the lower M:F ratio can be explained both by the lower number of declared MSM (less than 20\%) and by the higher number of women diagnosed with syphilis during pregnancy/birth compared with other countries (13).

The Centers for Disease Control and Prevention, Atlanta, USA, published a study in which syphilis patients were more frequently residing in urban areas while Thomas et al observed a higher concentration of syphilis cases in rural areas in North Carolina, USA $(15,16)$. The patients with syphilis in our study came mainly from urban areas $(67.42 \%)$, where internet use is more accessible, and sexual activity is more intense, with multiple partners, sometimes of the same sex and with condomless sex.

Over three-fourths of our patients with syphilis were aged between 15 and 45 years (76.52\%), with a maximum incidence between 15 and 30 years (4.1 cases per 100,000 inhabitants). The disease's maximum incidence in both women and men was between 20 and 24 years, according to our National Institute of Public Health (NIPH). In Europe, there is a maximum of cases in the age groups $25-34$ years $(16 / 100,000)$ and $35-44$ years
$(13 / 100,000)(17)$. All data confirm the maximum incidence of syphilis in the age groups with more intense sexual activity.

Analyzing the degree of schooling of our syphilis patients in the last decade, we noted that $51.01 \%$ of the patients had an educational level of grade 9-12. We found that many patients with syphilis had a medium level of education (grade 9-12) $(51.01 \%)$ compared to those with no or little education (0 or grades 1-4) (13.14\%), where we would have expected a higher incidence of the disease.

Analyzing the marital status in our group of patients, we noted that patients with syphilis were unmarried $(41.92 \%)$ or exhibited live-in cohabitation, usually from a poor socioeconomic status and a low level of education (grades 5-8) (22.22\%). It should be noted that about $1 / 4$ of patients $(27.27 \%)$ contacted the disease during marriage through extramarital affairs. Worldwide, poor socioeconomic status is a recognized risk factor, indirectly correlated with the syphilis prevalence, favoring some sexual behaviors (ex. condomless sex), without awareness of the possible consequences (17).

The distribution of the patients by disease stages was as follows: latent (early, 35.61\%; late, 32.32\%), secondary $(18.43 \%)$, primary $(11.11 \%)$, tertiary $(1.77 \%)$ and congenital syphilis $(0.76 \%)$. In our country, most cases of syphilis are early latent forms (12). Inexplicably, in our study, the tertiary syphilis was three times higher (1.77\%) compared to data from our NIPH.

This fact could suggest the possibility that tertiary syphilis may be underdiagnosed. Without the awareness of medical specialists in the field about the existence of these syphilis forms (that can occur silently or with neuropsychic pleomorphic manifestations), and specific investigations (cerebrospinal fluid serology for syphilis in dynamics), and without interdisciplinary collaboration, neurosyphilis could remain undiagnosed. We need to consider that tertiary syphilis can manifest itself after 20 years from the moment of infection, in individuals with low social and economic conditions (a vulnerable category that also exists in Romania), without any specific treatment. This could explain our result.

For the detection of syphilis in Romania, lifelong analysis filters are used (at marriage, in pregnancy, at blood donation or, a declaration of possible infected contacts by a patient already diagnosed with syphilis). In our study, over $50 \%$ of cases were diagnosed by an epidemiological investigation, respectively 
Table I. Demographic profile of the individuals diagnosed with syphilis over a period of 10 years (2009-2018) in Sibiu County, Romania (N=396).

\begin{tabular}{|c|c|c|c|}
\hline Characteristic & Subcategory & $\mathrm{n}(\%)$ & P-value \\
\hline Sex & $\begin{array}{l}\text { Male } \\
\text { Female }\end{array}$ & $\begin{array}{l}215(54.29) \\
181(45.71)\end{array}$ & $<0.001$ \\
\hline Area of residence & $\begin{array}{l}\text { Urban } \\
\text { Rural }\end{array}$ & $\begin{array}{l}267(67.42) \\
129(32.58)\end{array}$ & $<0.001$ \\
\hline Age groups (years) & $\begin{array}{l}\text { Under } 1 \text { year } \\
1-14 \text { years } \\
15-29 \text { years } \\
30-44 \text { years } \\
45-59 \text { years } \\
60-74 \text { years } \\
\text { Over } 75 \text { years }\end{array}$ & $\begin{array}{c}3(0.76) \\
1(0.25) \\
157(39.65) \\
146(36.87) \\
54(13.64) \\
33(8.33) \\
2(0.50)\end{array}$ & 0.001 \\
\hline Degree of schooling & $\begin{array}{l}\text { Without } \\
\text { Grades 1-4 } \\
\text { Grades 5-8 } \\
\text { Grades 9-12 } \\
\text { Higher education }\end{array}$ & $\begin{array}{r}18(4.55) \\
34(8.59) \\
88(22.22) \\
202(51.01) \\
54(13.64)\end{array}$ & $<0.001$ \\
\hline Marital status & $\begin{array}{l}\text { Married } \\
\text { Unmarried } \\
\text { Divorced } \\
\text { Widower } \\
\text { Concubinage }\end{array}$ & $\begin{array}{c}108(27.27) \\
166(41.92) \\
22(5.56) \\
12(3.03) \\
88(22.22)\end{array}$ & $<0.001$ \\
\hline Occupation & $\begin{array}{l}\text { Unemployed } \\
\text { Worker } \\
\text { Professional } \\
\text { Retiree } \\
\text { Driver } \\
\text { Others }\end{array}$ & $\begin{array}{c}128(32.32) \\
137(34.60) \\
45(11.36) \\
44(11.11) \\
9(2.27) \\
33(8.33)\end{array}$ & $<0.001$ \\
\hline
\end{tabular}

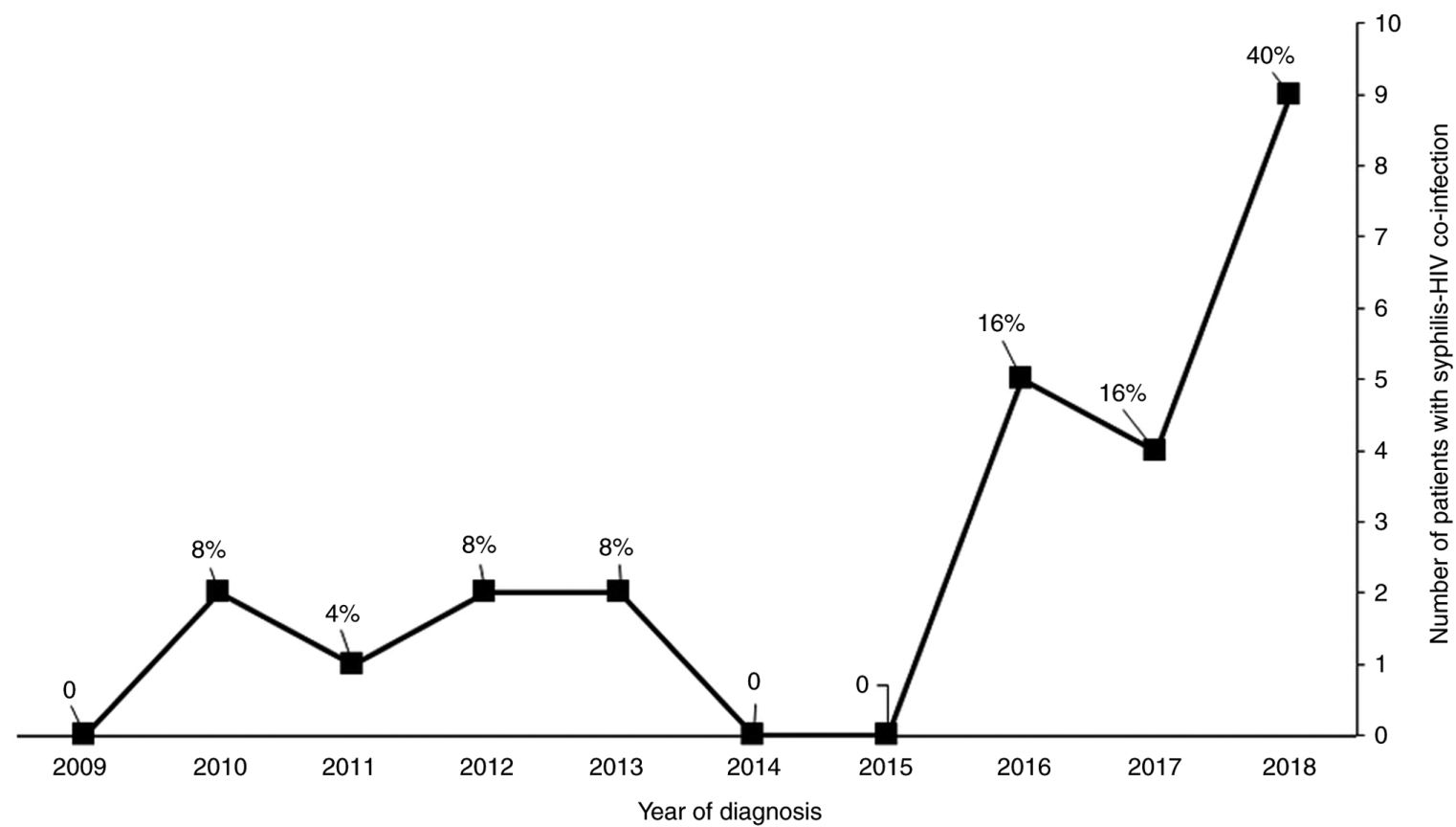

Figure 2. Ascending progressive distribution of syphilis-HIV co-infection over a period of 10 years (2009-2018) in Sibiu County. 
Table II. The therapy followed by our syphilis patients over a period of 10 years (2009-2018).

\begin{tabular}{lccccc}
\hline Year & $\begin{array}{c}\text { No of } \\
\text { patients }\end{array}$ & $\begin{array}{c}\text { Classic schemes } \\
\text { (Benzathine-penicillin } \\
\text { G/penicillin) } \\
\mathrm{n}(\%)\end{array}$ & $\begin{array}{c}\text { Cephalosporins } \\
\text { Cefuroxime/ceftriaxone) } \\
\mathrm{n}(\%)\end{array}$ & $\begin{array}{c}\text { Macrolides } \\
\text { (Azithromycin/ } \\
\text { erythromycin) } \\
\mathrm{n}(\%)\end{array}$ & $\begin{array}{c}\text { Doxycycline } \\
\mathrm{n}(\%)\end{array}$ \\
\hline 2009 & 78 & $78(100)$ & 0 & 0 & 0 \\
2010 & 49 & $46(93.88)$ & $1(2.04)$ & $2(4.08)$ & 0 \\
2011 & 30 & $27(90.00)$ & $1(3.33)$ & 0 & $2(6.67)$ \\
2012 & 38 & $36(94.74)$ & 0 & 0 & 0 \\
2013 & 23 & $21(91.30)$ & $2(8.70)$ & 0 & 0 \\
2014 & 21 & $17(80.95)$ & $4(19.05)$ & 0 & 0 \\
2015 & 35 & $30(85.71)$ & $5(14.29)$ & 0 & 0 \\
2016 & 30 & $25(83.33)$ & $5(16.67)$ & 0 & $7(17.07)$ \\
2017 & 41 & $16(39.02)$ & $18(43.90)$ & & $7(15.69)$ \\
2018 & 51 & $7(13.73)$ & $29(56.86)$ & & $7(13.73)$ \\
\hline
\end{tabular}

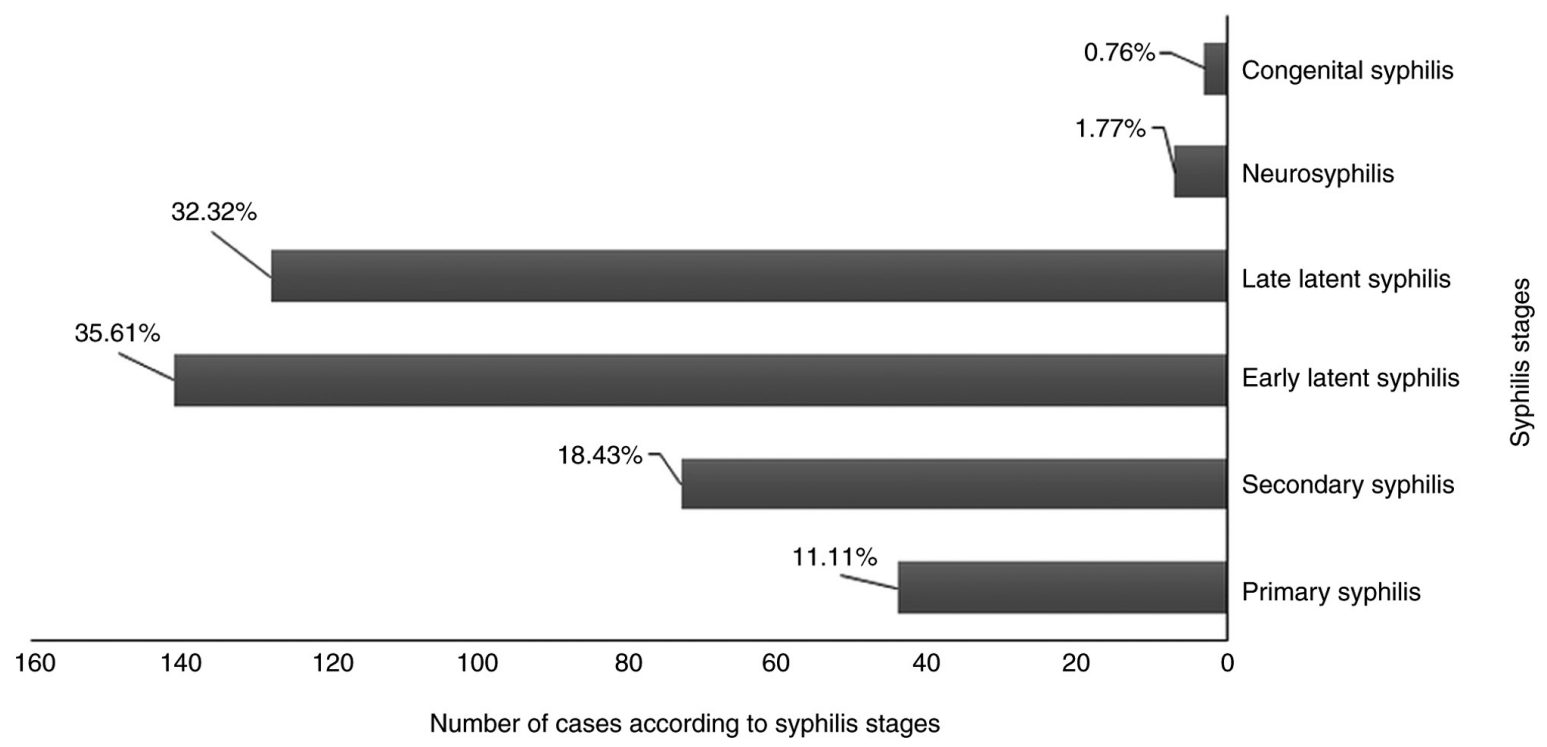

Figure 3. Distribution of syphilis cases according to the clinical stage (at the time of diagnosis).

by investigating all of the declared contacts of a new case of syphilis $(39.65 \%)$ and by mandatory testing of pregnant women $(14.39 \%)$. The high percentage of syphilis cases diagnosed during pregnancy or at birth is concerning; this category of patients exposing their fetus to the transplacental transmission of the infection, with the possibility of developing congenital syphilis in newborns. The epidemiological control of the syphilis contacts is significant in order to stop the transmission of the disease (through unidentified and untreated infected contacts). The declaration of all contacts by the infected person is essential for breaking the epidemiological chain in syphilis. From the group of 396 patients diagnosed with syphilis, we identified 276 contacts through epidemiological investigation, of which 183 were identified $(66.30 \%)$. The unidentified individuals were accidental sexual contacts with unknown persons from the country and/or from abroad.

Syphilis in pregnant women is an extremely sensitive topic for any society. The goal of the national health programs is to reduce this category of patients to 0. In 2009 and 2018, we analyzed the percentage of syphilis positivity in tested pregnant women. The percentage varied between $1.87 \%$ and $1.11 \%$, the average being $1.82 \%$ per decade, except for 2011 (a peak at $5.02 \%$ ). Given the possibility that the pregnant woman's syphilis evolved to congenital syphilis (with the transmission of the infection from mother to fetus), the presented data are alarming.

From January 2009 to December 2018, out of the 30,000 newborns in Sibiu County, 3 cases of congenital syphilis were diagnosed, this representing $0.76 \%$ of all cases of syphilis. At the national level (in 2016), there were 6 cases declared. Of all the congenital syphilis cases declared in Europe between 2007 and 2017, 11\% were from Romania (12). Also, in Romania, the rate of congenital syphilis decreased from 12.1 cases/100,000 newborns in 2007 to 3 cases/100,000 newborns in 2017 (12). Internationally, by 2018, 11 countries have declared that they have eliminated the transplacental transmission of luetic 
infection, Belarus and Moldova being the ones from Europe (7). On the other hand, the incidence of congenital syphilis in Romania is higher than other regions of Europe $(0.4 / 100,000$ newborns) and Southeast Asia (0.9/100,000 newborns) and lower than those found in Africa (48.9/100,000 newborns) and some regions of America (22.8/100,000 newborns) (7).

Pregnancy monitoring is accomplished through primary care. The mother's responsibility to perform luetic serology in the first and the last trimester of pregnancy and the pregnant woman's education and her partner regarding healthy sexual behavior are useful tools for preventing syphilis. In addition, pregnant women with syphilis require a specific therapy that needs to be initiated as early as possible to reduce the risk of congenital syphilis.

In the last 2 decades, in Europe, the increased number of MSM has been reflected in the incidence of syphilis in association with HIV infection, with a higher rate in urban areas. Also, drug use and promiscuous life-style are correlated with the statistical increase in syphilis-HIV co-infection cases. In Romania, the number of MSM and extramarital relationships, with unprotected contacts, with unknown people has increased in the last years. In our study, over the 10-year period, the syphilis-HIV coinfection was identified at $6.31 \%$ of the cases, with a maximum of cases in 2018 (40\%). From this category of patients, $56 \%$ were MSM. The presented results urge us to relaunch national programs for the prevention of sexually transmitted diseases. We need to disseminate sexual health information to risk groups and the general population, especially to young people, who have a higher risk of contracting a sexually transmitted disease.

In our study, the syphilis treatment scheme was in accordance with the Romanian Health Ministry, adapted to each stage of the disease. Alternative therapies (ceftriaxone, doxycycline, azithromycin) are recommended to be used only in case of allergy to penicillin. In 2009, all patients received benzathine-penicillin G treatment (BPG) (primary, secondary, late, and early latent syphilis) or penicillin (congenital syphilis, neurosyphilis, or pregnant women with syphilis). Since 2017, due to the lack of BPG in the Romanian pharmaceutical market, the chosen second line of treatment was cephalosporins (in 2018,56.86\% of the cases were treated with cefuroxime or ceftriaxone).

Out of the 396 syphilis patients, 172 were lost to follow-up $(43.43 \%)$. We determined the serologic treatment response (STR) for 224 patients after 6 months (primary, secondary, and congenital syphilis) and 1 year from diagnosis (latent and tertiary syphilis). We consider that STR was achieved when VDRL or RPR had a $\geq 4$-fold decline (18). We compared the STR obtained after BPG/penicillin (56.32\%) vs. cephalosporins $(61.11 \%)$ or doxycycline therapy $(72.73 \%)$, and we observed that this was relatively similar in all treatment groups. Out of all of the groups, treatment with azithromycin/erythromycin had the lowest STR (50\%). For primary, secondary, and early latent syphilis, Cao et al demonstrated that STR to cephalosporins vs. BPG was approximately similar (90.2 vs. 78\%) after 6 months (19).

In conclusion, the incidence of syphilis from January 2009 to December 2018 in Sibiu County showed a downward trend, following the national data, but in antithesis with the international data. Our county's syphilis patient demographic profile is male, aged 15-30 years, from the urban area, with an educational level of grades 9-12, unmarried, worker or unemployed. During this period, our county's progressive increase in syphilis-HIV co-infection and neurosyphilis cases is concerning. Due to the lack of the pharmaceutical product, first-line therapy with benzathine-penicillin G/penicillin or penicillin has been replaced in the last 3 years with the 2 nd line of treatment (cephalosporins, doxycycline, macrolides). The serologic treatment response was relatively similar to BPG/penicillin, cephalosporins, and doxycycline, but lower for macrolides.

\section{Acknowledgements}

Not applicable.

\section{Funding}

No funding was received.

\section{Availability of data and materials}

All the data are available from the corresponding author.

\section{Authors' contributions}

GMI conducted the research creation and design, analysis and interpretation of patient data, manuscript drafting and critical revision of the manuscript for important intellectual content. MR conducted the design and critical revision of the manuscript for important intellectual content. Both authors read and approved the manuscript and agree to be accountable for all aspects of the research in ensuring that the accuracy or integrity of any part of the work are appropriately investigated and resolved.

\section{Ethics approval and consent to participate}

The study was approved by the Ethics Committee of the County Emergency Hospital of Sibiu, Romania (no. 25413/05.12.2019).

\section{Patient consent for publication}

Not applicable.

\section{Competing interests}

The authors declare no competing interests.

\section{References}

1. Danescu SA, Szolga B, Georgiu C, Surcel A and Senila SC: Unusual manifestations of secondary syphilis: Case presentations. Acta Dermatovenerol Croat 26: 186-188, 2018.

2. Rotaru M, Maniu H, Lisandru M and Iancu G: Diagnostic difficulties in secondary syphilis associated with psoriasis vulgaris-clinical case. Dermatovenerologia 61: 249-256, 2016.

3. Parker SR, Correnti C, Sikora K and Parker DC: Seronegative syphilis: Another case for the great imitator. Int J Infect Dis 18: 104-105, 2014.

4. Oanta A and Irimie M: Syphilitic balanitis of follmann. Int J Dermatol 53: 830-831, 2014. 
5. Etheridge T, Bowen RC, Raven M, Snow KB, Urban AW and Chang JS: Ocular syphilis: Clinical manifestations and treatment course. WMJ 118: 191-195, 2019.

6. Kojima $\mathrm{N}$ and Klausner JD: Un update on the global epidemiology of syphilis. Curr Epidemiol Rep 5: 24-38, 2018.

7. World Health Organization: Sexually Transmitted Infections (STIs). https://www.who.int/publications/data/gho/data/themes/ sexually-transmitted-infections. Accessed June 16, 2020.

8. Grad AI, Senila SC, Cosgarea R, Tataru AD, Vesa SC, Vica ML, Matei HV and Ungureanu L: Sexual behaviors, attitudes, and knowledge about sexually transmitted infections: A cross-sectional study in Romania. Acta Dermatovenerol Croat 26: 25-32, 2018.

9. Centers for Disease Control and Prevention: Syphilis.https://www. cdc.gov/std/stats17/syphilis.htm. Accessed July 2, 2020.

10. Karp G, Schlaeffer F, Jotkowitz A and Riesenberg K: Syphilis and HIV co-infection. Eur J Intern Med 20: 9-13, 2009.

11. International Union Against Sexually Transmitted Infections 2019 European Congress: Abstract Book. www.conference-expert. eu/en/iusti2019/en/abstract-book/1. Accessed June 2, 2020

12. European Centre for Disease Prevention and Control: Syphilis and congenital syphilis in Europe-A review of epidemiological trends (2007-2018) and options for response. https://www.ecdc.europa.eu/sites/default/files/documents/syphilisand-congenital-syphilis-in-Europe.pdf. Accessed February 18, 2021.
13. European Centre for Disease Prevention and Control: Syphilis notifications in the EU/EEA up by $70 \%$ since 2010. https:// www.ecdc.europa.eu/en/news-events/syphilis-notifications-eueea70-2010. Accessed October 11, 2020.

14. Chen ZQ, Zhang GC, Gong XD, Lin C, Gao X, Liang GJ, Yue XL, Chen XS and Cohen MS: Syphilis in China: Results of a national surveillance programme. Lancet 369: 132-138, 2007.

15. Singh AE and Romanowski B: Syphilis: Review with emphasis on clinical, epidemiologic, and some biologic features. Clin Microbiol Rev 12: 187-209, 1999.

16. Thomas JC, Kulik AL and Schoenbach VJ: Syphilis in the South: Rural rates surpass urban rates in North Carolina. Am J Public Health 85: 1119-1122, 1995.

17. European Centre for Disease Prevention and Control: Syphilis and congenital syphilis in Europe-A review of epidemiological trends (2007-2018) and options for response. https://www.ecdc. europa.eu/en/publications-data/syphilis-and-congenital-syphilis-europereview-epidemiological-trends-2007-2018. Accessed June 28, 2020.

18. Brown DL and Frank JE: Diagnosis and management of syphilis. Am Fam Physician 68: 283-290, 2003.

19. Cao Y, Su X, Wang Q, Xue H, Zhu X, Zhang C, Jiang J, Qi S, Gong X, Zhu X, et al: A multicenter study evaluating Ceftriaxone and Benzathine Penicillin $\mathrm{G}$ as treatment agents for early syphilis in Jiangsu, China. Clin Infect Dis 65: 1683-1688, 2017. 